Stereotactic and Functional

Neurosurgery
Stereotact Funct Neurosurg 2011;89:48-55

DOI: $\underline{10.1159 / 000323340}$
Received: June 27, 2010

Accepted after revision: December 2, 2010

Published online: January 19, 2011

\title{
Analysis of the Time Course of the Effect of Subthalamic Nucleus Stimulation upon Hand Function in Parkinson's Patients
}

\author{
Ben Waldau Daniel A. Clayton Lynne B. Gasperson Dennis A. Turner \\ Department of Surgery, Division of Neurosurgery, Duke University Medical Center, Durham, N.C., USA
}

\section{Key Words}

Parkinson's disease $\cdot$ Deep brain stimulation $\cdot$ Subthalamic nucleus $\cdot$ Movement time $\cdot$ Reaction time $\cdot$ Velocity $\cdot$

Hand function

\begin{abstract}
Background: Deep brain stimulation (DBS) of the subthalamic nucleus (STN) as treatment for Parkinson's disease has been in use for more than a decade, yet the immediate effect of stimulation upon movement parameters is not well characterized. Objective: The goal of the current study is the identification of the best time point to test hand function after programming DBS devices. Methods: Reaction time, movement time and velocity were measured at multiple time points with a movement-sensitive glove after the deep brain stimulator had been turned on or off, during 'off medication' conditions. Results: Velocity, movement time and reaction time worsened significantly in the first $20 \mathrm{~min}$ after the deep brain stimulator had been turned off. A 'plateau effect' after 20 min was not observed. Initiation of stimulation led to immediate significant increases in movement time and velocity and to a lesser degree a decrease in reaction time. Patients performed more inconsistently over time after onset of stimulation compared to stimulation withdrawal. In-
\end{abstract}

traoperative testing showed an immediate improvement in velocity after placement of the STN deep brain stimulator. Conclusion: Movement time and velocity already reach their peak changes within 20 min after the deep brain stimulator has been reprogrammed, and therefore, this time point may be used to test the maximal clinical effect of stimulation.

Copyright $\odot 2011$ S. Karger AG, Basel

\section{Introduction}

Deep brain stimulation (DBS) of the subthalamic nucleus (STN) is an accepted procedure for symptoms of Parkinson's disease (PD) [1]. Targeting of the STN at our institution is based on single-unit recording, with kinesthetic neurons being localized within a tract through the STN [2]. A promising tract is identified by significant bursting activity, a high level of background neural activity, large units, a frequency of commonly $50-80 \mathrm{~Hz}$ and a kinesthetic response to contralateral movement, for at least $5.0-6.0 \mathrm{~mm}$ in depth. Subsequently, the treatment electrode is placed and test stimulation is performed. Assessing the immediate response to stimulation is largely based upon the patient's subjective sense of improvement and clinical motor evaluation. Postoperative program-

\section{KARGER}

Fax +4161306 1234 E-Mail karger@karger.ch www.karger.com (c) 2011 S. Karger AG, Basel

1011-6125/11/0891-0048\$38.00/0

Accessible online at:

www.karger.com/sfn
Ben Waldau, MD

DUMC, Box 3807

Durham, NC 27710 (USA)

Tel. +1 919684 3271, Fax +1 9196817973

E-Mail ben.waldau@duke.edu 
Table 1. STN stimulation settings of patients studied postoperatively

\begin{tabular}{lllr}
\hline Patient & \multicolumn{2}{l}{ Left STN } & \multicolumn{2}{l}{ Right STN } \\
\hline 1 & $2-/ 3-/ \mathrm{C}+2.4 \mathrm{~V} / 60 \mu \mathrm{s} / 185 \mathrm{~Hz}$ & $2-/ 3-/ \mathrm{C}+$ & $2.4 \mathrm{~V} / 90 \mu \mathrm{s} / 185 \mathrm{~Hz}$ \\
2 & $1-/ 2-/ \mathrm{C}+2.1 \mathrm{~V} / 60 \mu \mathrm{s} / 185 \mathrm{~Hz}$ & $1-/ 2-/ \mathrm{C}+$ & $1.8 \mathrm{~V} / 60 \mu \mathrm{s} / 185 \mathrm{~Hz}$ \\
3 & $5-/ 6-/ \mathrm{C}+2.5 \mathrm{~V} / 60 \mu \mathrm{s} / 185 \mathrm{~Hz}$ & $1-/ 2-/ \mathrm{C}+$ & $2.5 \mathrm{~V} / 60 \mu \mathrm{s} / 185 \mathrm{~Hz}$ \\
4 & $0-/ 1-/ 2+5 \mathrm{~V} / 90 \mu \mathrm{s} / 185 \mathrm{~Hz}$ & $0-/ 1-/ 2+$ & $5 \mathrm{~V} / 90 \mu \mathrm{s} / 185 \mathrm{~Hz}$ \\
5 & $2-/ 3-/ \mathrm{C}+2.4 \mathrm{~V} / 60 \mu \mathrm{s} / 185 \mathrm{~Hz}$ & $1-/ 2-/ 3-/ \mathrm{C}+1.4 \mathrm{~V} / 60 \mu \mathrm{s} / 185 \mathrm{~Hz}$ \\
6 & $2-/ 3-/ \mathrm{C}+2.0 \mathrm{~V} / 90 \mu \mathrm{s} / 185 \mathrm{~Hz}$ & $2-/ 0+$ & $2.9 \mathrm{~V} / 90 \mu \mathrm{s} / 185 \mathrm{~Hz}$ \\
\hline
\end{tabular}

ming for PD patients would also benefit from the development of more objective measures of bradykinesia and rigidity. Because objective motor parameters in monkeys administered 1-methyl-4-phenyl-1,2,5,6-tetrahydropyridine suggest that complex motor tasks (e.g. goal-directed behavioral tasks) might better represent the response to relief of bradykinesia than simple, repetitive motions [3, 4], this type of task may have advantages in assessing programming benefit. Immediate changes in motor excitability have been shown to occur with DBS [5]. While the relief of rigidity can be measured by EMG [6] and is thought to be quite rapid upon stimulation induction [7], the improvement in bradykinesia is measured by motor tasks and may plateau only after hours [8].

The aim of the current study was to characterize the time course of bradykinesia as well as akinesia after STN DBS was initiated or withdrawn. Reaction time and velocity were measured as surrogate markers of akinesia and bradykinesia, respectively. However, akinesia and bradykinesia may show a more complex clinical picture than just reaction time and velocity, which makes their measurement artificial in this study.

We hypothesize that effective STN DBS leads to a significant improvement in movement velocity compared to DBS off conditions, but that the time course of switching on and off the deep brain stimulator may vary considerably due to circuit and entrainment effects of stimulation of the STN and connected brain regions. To analyze this hypothesis, we chose a simple, video-based hand task, sufficiently robust so that awake patients during DBS procedures, as well as clinic patients, could rapidly and accurately perform the task.

\section{Methods}

This study was a prospective clinical trial to compare the behavioral performance of PD patients during 'off medication' conditions and controls. The Duke University Institutional Review
Board approved all procedures. Three forms of testing were performed: (1) patients undergoing DBS surgery were tested intraoperatively; (2) Parkinson's patients were tested off medications at least 6 months after STN DBS had been performed, and (3) agematched controls were tested.

Intraoperative analyses were performed on patients before and after the placement of STN DBS leads $(\mathrm{n}=5 ; 7$ sides) and ventral intermediate nucleus (VIM) DBS leads ( $\mathrm{n}=3 ; 5$ sides) to assess the immediate benefit and side effects of electrical stimulation. The zona incerta was not stimulated after VIM DBS lead placement since no insertions passed the AC-PC line.

The virtual reality stretch glove task was performed by a separate group of postoperative patients in the clinic setting. Preoperatively, all of these patients had frequent daily Sinemet dosing (average 7.8 doses/day), severe dyskinesias, on/off fluctuations and persistent rigidity, severely limiting their daily activities; 3 also had severe tremor. All patients had an excellent response to Sinemet preoperatively but only a very short duration of action (hence necessitating the frequent dosing). Average age at the time of surgery was 61.5 years, and the preoperative duration of $\mathrm{PD}$ averaged 15 years. Postoperatively, all of these major PD symptoms improved, with no dyskinesias, no on/off fluctuations, no tremor and a slight decrease in Sinemet dosing (to 4.7 doses/day). All patients were studied $>2$ years after their surgery and showed stable DBS programming (active contacts and settings are summarized in table 1). The studied patients were all much more active postoperatively than preoperatively. These patients essentially represent the 'ideal' response to surgery since almost all their motor symptoms resolved completely and they experienced much greater 'on' time throughout the day. The patients were evaluated in two separate clinic visits to test reaction time, movement time and velocity after the deep brain stimulator was turned off ( 9 sides) and after the deep brain stimulator was turned on (10 sides). Both tests were performed at least $12 \mathrm{~h}$ after cessation of all PD medications. The half-life of regular Sinemet is approximately $2 \mathrm{~h}$. However, the authors cannot exclude residual medication effects during testing.

Lastly, control volunteer individuals $(n=5)$, age-matched for the PD patients, were enrolled, who also underwent sessions for comparison to the PD patients. Each subject (both controls and PD patients) was scheduled for two visits at least 7 but no more than 60 days apart.

All PD subjects had been implanted with a bilateral STN deep brain stimulator and were doing well with stable programming of their DBS device. Implantation was performed using standard MRI-based framed stereotactic techniques using microelectrode 


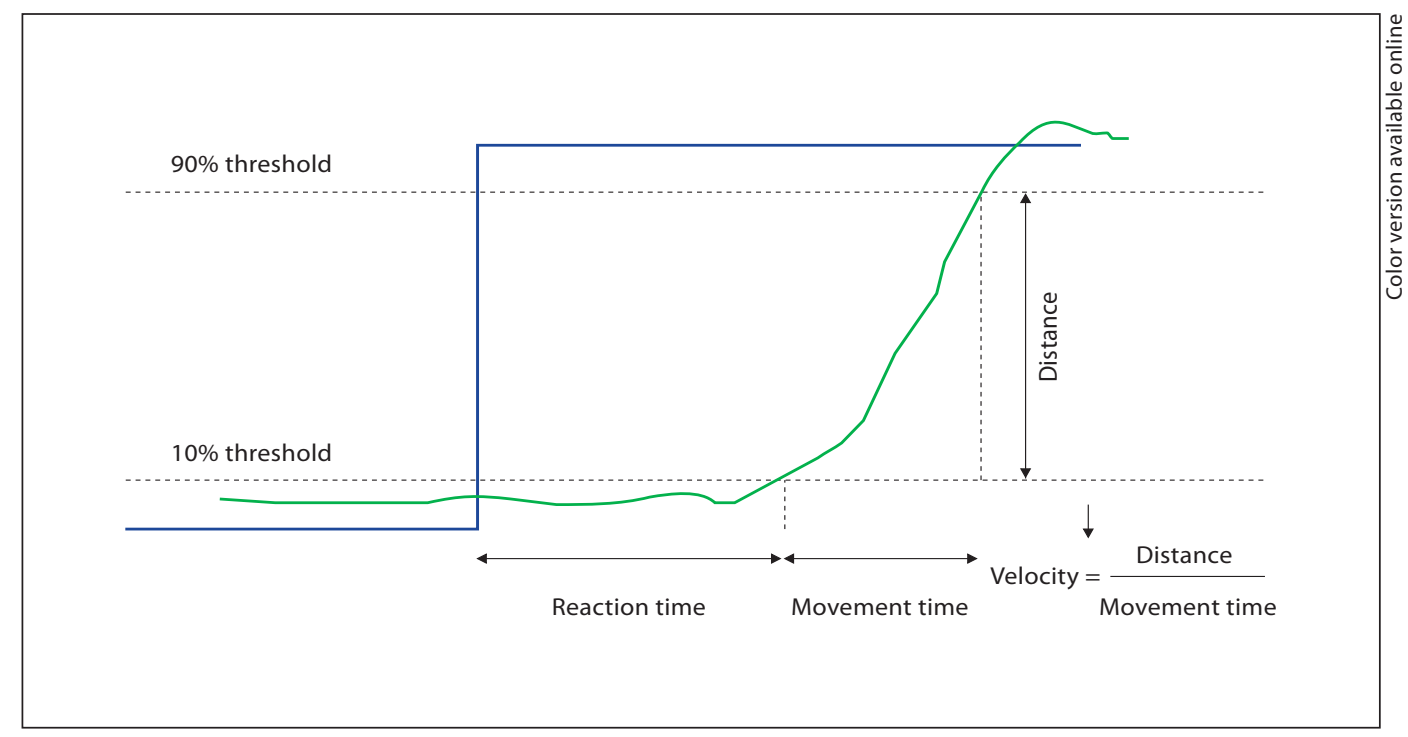

Fig. 1. Illustration of the measurements derived from the video game. Reaction time was defined as the time needed to cross a threshold of $10 \%$ of the difference to the new target. Movement time was the time between crossing of the $10 \%$ threshold until crossing of the $90 \%$ threshold. Velocity was computed as the 'distance' the hand moved divided by the movement time.

recordings and postoperative CT imaging to confirm appropriate placement. All subjects were instructed to stop all anti-Parkinson medications at least $12 \mathrm{~h}$ prior to the visit. Patients were alternately assigned to 2 groups; one group was instructed to turn their deep brain stimulator off the night before the first visit and the other group before the second visit. The examiner first confirmed the 'off' medication status with the subject, then noted the 'on' or 'off' status of the DBS unit. Assignments to either group were made at random. The second visit allowed testing in the opposite condition. These two experimental conditions allowed us to examine the time course of both stimulus onset and stimulus withdrawal in the same subjects, but not consecutively, and in the absence of interfering medications.

The patients were placed in a sitting position in front of a video screen as described previously [9]. A virtual reality stretch glove on each hand continuously measured the position and motion of the fingers and hand, at $100-\mathrm{Hz}$ updates. The glove provided accurate time information, but movement position was relative and could only be scaled between values of 0 (fully closed fist) and 1 (fully open hand with fingers outstretched). The patient was then instructed to adjust the hand and finger position to reach a new target level on the video screen as rapidly as possible using their hand position, once the target was presented. A visual representation of hand position was provided to the patient as the location of a vertical bar on a video monitor. The motor task required the patient to match the bar to the target, by adjusting the position of the virtual reality glove. Once at a target level, the patient was required to hold that hand position for a short, random period $(0.5-1.0 \mathrm{~s})$ before the target level changed. There was no alerting for the next target level. Reaction time and maximum velocity of hand movement could then be calculated for each trial, and an average was obtained to characterize the patient's performance.

Reaction time was defined as the time needed to cross a threshold of $10 \%$ of the difference to the new target (fig. 1). Movement time was the time between crossing of the $10 \%$ threshold until crossing of the $90 \%$ threshold of hand position as the new target was reached. Velocity was computed as the 'distance' the hand moved divided by the movement time. Note that the distances actually moved were in the $1-$ to $2-\mathrm{cm}$ range, but these were then scaled for each patient, hence no absolute distances could be measured from the data, and all velocity measurements are 'relative'. In this paradigm, movement time is also representative of velocity since the distances were small and nearly the same from patient to patient.

A session consisted of playing the video game until 20 hits were achieved. A test consisted of 4 sessions, alternating the dominant and nondominant hand, with a rest of at least $1 \mathrm{~min}$ between the end of one session and the start of the next. At the start of the visit, the subject was allowed to practice the game with both hands until he/she felt comfortable. During this time, the operator adjusted the game so that the subject could easily move the cursor the full length of the screen. When the subject was ready for testing, a baseline test was recorded. Immediately afterwards, the deep brain stimulator was either switched on or off. Additional tests were recorded at approximately 5, 10, 15, 30 and $60 \mathrm{~min}$. Testing times could not be followed strictly in some Parkinsonian patients due to fatigue or poor performance; some patients fell considerably behind the testing schedule so that the testing period reached $100 \mathrm{~min}$ after deep brain stimulator programming. Therefore, the exact time of the tests was recorded so that each session could later be allocated to the correct time window. 


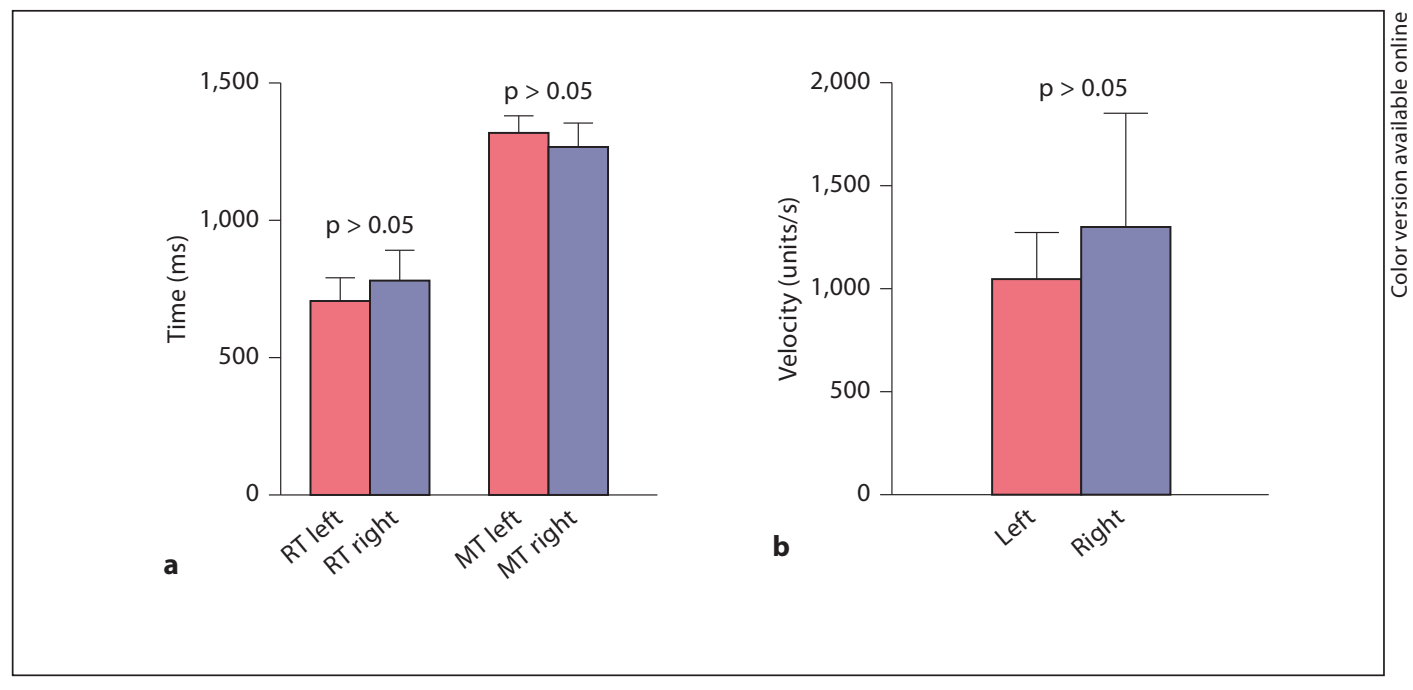

Fig. 2. Comparison between the right hand and the left hand showed that there was a strong trend towards better performance for reaction time (RT) and movement time (MT) (a) as well as velocity (b) with the right hand.

\begin{abstract}
All patient data were maintained in an extensive database, for the calculation of the derived variables, coded by patient number (with no other identifiers). All session data were reviewed and then averaged for each test time point. The testing period was subdivided into 5 time windows ( $\leq 20,21-40,41-60,61-80$ and $81-100 \mathrm{~min}$ ). All tests performed in a specific time interval were averaged and the standard deviation was calculated. A paired Student's two-tailed t test was performed to compare each time interval and the baseline in order to test for significant changes in reaction time, movement time and velocity.
\end{abstract}

\section{Results}

A summary of the results obtained from the control subjects is presented in figure 2. No significant hand bias was detected; however, there was a strong trend towards better performance with the right hand. Control subjects had an average choice reaction time of $741.8 \mathrm{~ms}$, compared to $1,003.4 \mathrm{~ms}$ for Parkinsonian patients while their stimulator was turned on at baseline and 1,461.6 ms while their stimulator was turned off. Control subjects had an average movement time of 1,296.6 ms, compared to $1,799.6 \mathrm{~ms}$ in PD patients with their deep brain stimulator switched on at baseline and 2,002.5 ms with their stimulator switched off. Finally, controls showed an average velocity of $1,162.6$ units/s. Parkinsonian patients had a velocity of $1,188.6$ units/s with their stimulators turned on at baseline and 800.3 units/s when their stimulators were turned off.
Turning the stimulator off led to significant worsening of reaction time, movement time and velocity (fig. 3a, b). Movement time increased in the first $20 \mathrm{~min}$ from an average of $1,799.6$ to $2,902.3 \mathrm{~ms}$, or by $62 \%$. Meanwhile, the reaction time increased from $1,003.4$ to $1,311.9 \mathrm{~ms}$, or by $31 \%$. The velocity dropped within the first $20 \mathrm{~min}$, from $1,188.6$ to 910.0 units/s, a $23 \%$ decrease. All changes in movement time, reaction time and velocity in the first 20 min proved to be significant. However, none of the 3 testing parameters showed a trend for further worsening over the remaining time period after the initial decline, but rather remained steady at the same level of performance.

Turning the stimulator on significantly decreased the movement time from $2,002.5$ to $1,525.3 \mathrm{~ms}$, or by $24 \%$ in the first $20 \mathrm{~min}$ (fig. 4a). The reaction time decreased in the same time interval by $17 \%$, from $1,461.6$ to $1,210.8 \mathrm{~ms}$, which did not reach significance (fig. 4a). However, the improvement in reaction time did reach significance during testing once in the 41- to 60 -min window. Velocity significantly increased from 800.3 to 991.011 units/s, or by $19 \%$ in the first $20 \mathrm{~min}$ (fig. $4 \mathrm{~b}$ ). Over the entire testing period, the patients showed a more inconsistent picture of performance with the deep brain stimulator turned on than after the deep brain stimulator was switched off. There was a general trend for an increase in velocity and a decrease in reaction time and movement time.

Intraoperative testing showed a significant increase in velocity after both STN and VIM deep brain stimulator 


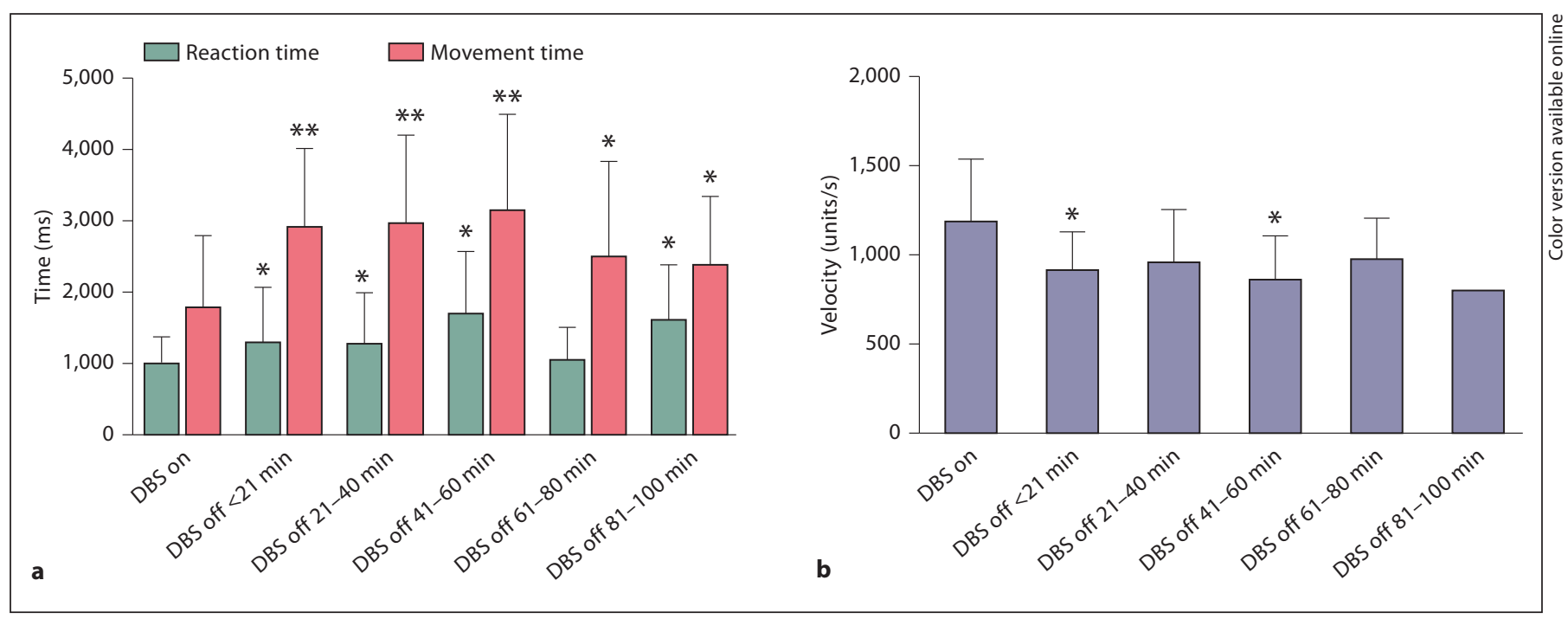

Fig. 3. Turning the deep brain stimulator off led to significant worsening of movement time and reaction time (a) as well as velocity (b) within the first $20 \mathrm{~min}$. There was no significant trend towards further decline over the rest of the testing period. ${ }^{*} \mathrm{p}<0.05,{ }^{* *} \mathrm{p}<0.01$ : compared to DBS on.

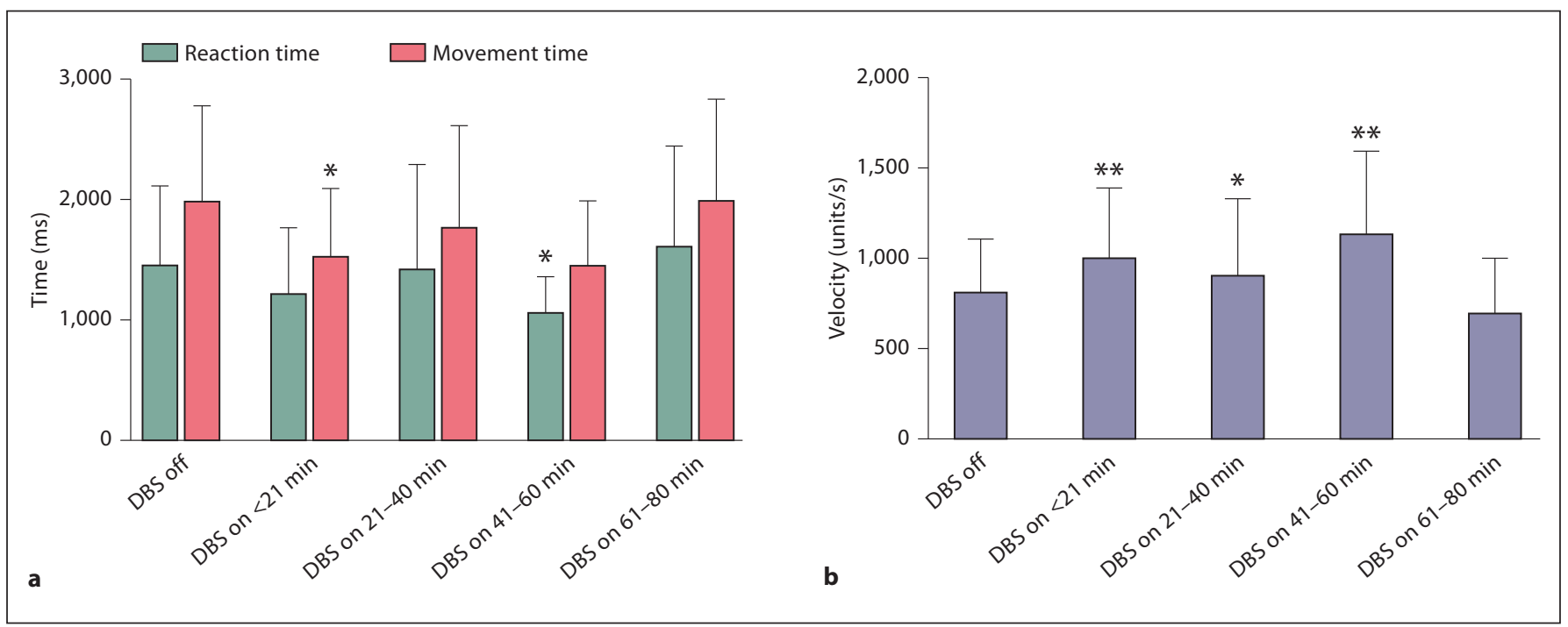

Fig. 4. Turning the deep brain stimulator on led to a significant improvement in movement time (a) and velocity (b) within the first $20 \mathrm{~min}$. There was also an improvement in reaction time (a); however, this only reached significance after 41-60 min. Overall, patient performance was more inconsistent over time than when the stimulator was switched off. This may be due to patient fatigue in the setting of prolonged testing or due to the need for stabilization of the stimulated circuits. ${ }^{*} \mathrm{p}<0.05,{ }^{* *} \mathrm{p}<0.01$ : compared to DBS off. 


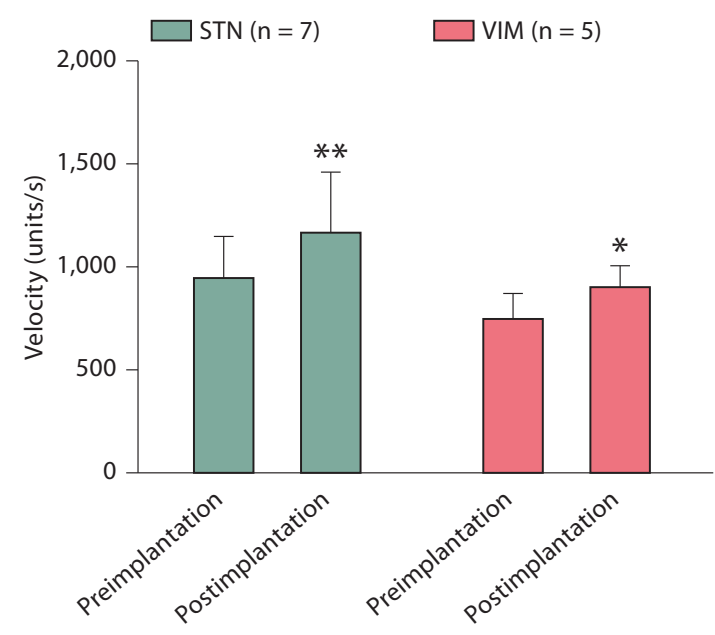

Fig. 5. Intraoperative testing showed a significant increase in velocity after both STN and VIM deep brain stimulator placement. The increase in velocity was highly significant for STN stimulator placement $\left({ }^{* *} \mathrm{p}<0.01\right)$ but barely reached significance for VIM placement $\left({ }^{*} \mathrm{p}=0.0433\right)$.

placement (fig. 5). The velocity after STN deep brain stimulator placement improved from 932 to $1,177.3$ units/s, or by $21 \%$. After VIM deep brain stimulator placement, the velocity increased from 751.4 to 894.9 units/s, or by $19 \%$. The increase in velocity was highly significant for STN deep brain stimulator placement $(\mathrm{p}<$ 0.01 ), but barely reached significance for VIM deep brain stimulator placement $(\mathrm{p}=0.0433)$.

\section{Discussion}

The goal of the current study was to elucidate the time course of the effect of STN stimulation upon hand function in Parkinsonian patients. In the past, paradigms for the analysis of bradykinesia and akinesia in $\mathrm{PD}$ patients involved tapping [10], wrist pronation-supination [4] or motor precision tasks [11], as well as Unified Parkinson's Disease Rating Scale (UPDRS) measurements [10]. We developed a motor precision task, which used a virtual reality stretch glove connected to a computer interface, for rapid and reliable collection of information to characterize the time course of the effect of stimulation on bradykinesia (movement time and velocity) and akinesia (reaction time).

Time Course of DBS Effect in Parkinson's Disease
We found that reaction time, movement time and velocity worsened significantly within $20 \mathrm{~min}$ after the deep brain stimulator had been switched off. After the initial drop in performance, there was no significant further decline; hence, withdrawing stimulation did not have any delayed effects on performance with respect to the parameters tested. An immediate significant effect on movement time and velocity and a delayed significant effect on reaction time were seen after the start of stimulation. Overall, movement time, reaction time and velocity showed a more inconsistent change in profile over time after starting stimulation than after withdrawal of stimulation. This may be due to a slow stabilization of the circuits involved with the stimulation effect as they become activated.

Other studies have also shown that a change in DBS has an early effect on performance, though delayed effects may also occur over several days. Wenzelburger et al. [12] showed that withdrawal of STN DBS results in a decline in UPDRS motor scores within the first $30 \mathrm{~min}$. Levy et al. [4] also used the time interval of $30 \mathrm{~min}$ and showed that turning the deep brain stimulator on or off leads to significant changes in wrist pronator-supinator movements when performed together with a tapping task. Lopiano et al. [13] found that movement time increased progressively after stimulation withdrawal, reaching a plateau after about $30 \mathrm{~min}$, which then lasted for the subsequent $2 \mathrm{~h}$ of observation time. Finally, Temperli et al. [8] showed that bradykinesia, rigidity, tremor and UPDRS motor scores drop off sharply after the stimulator has been switched off for $5 \mathrm{~min}$. However, their findings of an immediate $75 \%$ decrease in performance which is followed by a plateau after $4 \mathrm{~h}$ could not be reproduced by us. Reaction time, movement time and velocity did not consistently show any further decline after the initial immediate decay in our study. Measures of clinical ratings (e.g. UPDRS) may be measuring different types of performance than more specific data on bradykinesia and actual movement, which may explain the discrepancy in these results.

Movement time showed significant changes within 20 min of starting stimulation, whereas reaction time only showed significant decreases after stimulation for 41-60 min. It has been previously reported that stimulation significantly improves movement time and dexterity, but has little or no effect on reaction time [14]. However, other groups have found that STN stimulation does improve simple reaction time and choice reaction time as well [15, 16]. Our results show that both movement time and reaction time improve, but movement time improves more rapidly and significantly than reaction time. This is con- 
sistent with earlier results which showed that movement time is a better predictor of DBS parameters than reaction time.

Velocity also showed significant increases with stimulation as well as significant decreases with stimulation withdrawal, almost immediately after the stimulation was discontinued. Other reports have observed similar changes in velocity in a hand task, also with near-immediate improvement with stimulation onset $[13,17,18]$.

Intraoperative sessions confirmed that velocity in the hand motor task showed a highly significant improvement in patients almost immediately after placement of STN DBS leads. Thus, the virtual reality stretch glove connected to a computer interface may be useful as a rapid gauge to check appropriate lead placement in the intraoperative setting, complementing the patient's subjective sense of improvement. Intraoperative stimulation of the VIM in patients with essential tremor also led to a barely significant increase in velocity. The reason for this unexpected finding is not clear. Although there is a training effect with the virtual reality stretch glove, this effect did not reach significance in our control population (data not shown). Therefore, a training effect is less likely to explain this finding. Possibly, the decrease in tremor intensity with stimulation may enhance the patient's self-confidence to approach the target with higher velocity or with greater accuracy. However, the validity of measuring bradykinesia with the virtual reality stretch glove in PD patients comes into question if the task also leads to significant improvements in patients who do not suffer from bradykinesia.
In conclusion, onset and withdrawal of DBS lead to immediate changes in movement time, reaction time and velocity in Parkinsonian patients. A 'plateau effect' was not observed over time after stimulation was initiated or withdrawn. Thus, our results are most consistent with those of Lopiano et al. [13], who also did not observe a 'plateau effect' after more than $30 \mathrm{~min}$. Testing of PD patients may therefore be performed immediately after changes in stimulation parameters are made in the clinic or in the operating room, but it may require 20-30 min for full stabilization of the changes after programming. The limitations of the current study include the small number of patients studied, the study period of only 60 min and the possibility of lingering medication effects even after $12 \mathrm{~h}$ of discontinuation. Delayed effects may occur much later than $60 \mathrm{~min}$, as shown in previous studies [8]. During prolonged testing, patients showed a more inconsistent response in movement time and reaction time after the stimulation had been initiated. Further research is needed to investigate whether this finding is due to patient fatigue or due to a need of the effective stimulation response to stabilize over a period of hours.

\section{Acknowledgements}

This work was supported by Duke University Research Funds and Defense Advanced Research Projects Agency Broad Agency Announcement 01-42 Program Grant.

\section{References}

1 Benabid AL, Chabardes S, Mitrofanis J, Pollak P: Deep brain stimulation of the subthalamic nucleus for the treatment of Parkinson's disease. Lancet Neurol 2009;8:67-81.

-2 Shin M, Lefaucheur JP, Penholate MF, Brugieres P, Gurruchaga JM, Nguyen JP: Subthalamic nucleus stimulation in Parkinson's disease: Postoperative CT-MRI fusion images confirm accuracy of electrode placement using intraoperative multi-unit recording. Neurophysiol Clin 2007;37:457466.

-3 Camarata PJ, Parker RG, Park SK, Haines SJ, Turner DA, Chae H, Ebner TJ: Effects of 1-methyl-4-phenyl-1,2,5,6-tetrahydropyridine (MPTP)-induced hemiparkinsonism on the kinematics of a two-dimensional, multijoint arm movement in the rhesus monkey. Neuroscience 1992;48:607-619.
4 Levy R, Lang AE, Hutchison WD, Lozano AM, Dostrovsky JO: Simultaneous repetitive movements following pallidotomy or subthalamic deep brain stimulation in patients with Parkinson's disease. Exp Brain Res 2002;147:322-331.

5 Hanajima R, Ashby P, Lozano AM, Lang AE, Chen R: Single pulse stimulation of the human subthalamic nucleus facilitates the motor cortex at short intervals. J Neurophysiol 2004;92:1937-1943.

-6 Levin J, Krafczyk S, Valkovic P, Eggert T, Claassen J, Botzel K: Objective measurement of muscle rigidity in Parkinsonian patients treated with subthalamic stimulation. Mov Disord 2009;24:57-63.

7 Volkmann J, Herzog J, Kopper F, Deuschl G: Introduction to the programming of deep brain stimulators. Mov Disord 2002;17 (suppl 3):S181-S187.
-8 Temperli P, Ghika J, Villemure JG, Burkhard PR, Bogousslavsky J, Vingerhoets FJ: How do parkinsonian signs return after discontinuation of subthalamic DBS? Neurology 2003; 60:78-81.

-9 Patil PG, Carmena JM, Nicolelis MA, Turner DA: Ensemble recordings of human subcortical neurons as a source of motor control signals for a brain-machine interface. Neurosurgery 2004;55:27-35, discussion 35-38.

10 Taylor Tavares AL, Jefferis GS, Koop M, Hill BC, Hastie T, Heit G, Bronte-Stewart HM: Quantitative measurements of alternating finger tapping in Parkinson's disease correlate with UPDRS motor disability and reveal the improvement in fine motor control from medication and deep brain stimulation. Mov Disord 2005;20:1286-1298. 
11 Frankemolle AM, Wu J, Noecker AM, Voelcker-Rehage C, Ho JC, Vitek JL, McIntyre CC, Alberts JL: Reversing cognitivemotor impairments in Parkinson's disease patients using a computational modelling approach to deep brain stimulation programming. Brain 2010;133:746-761.

12 Wenzelburger R, Kopper F, Zhang BR, Witt K, Hamel W, Weinert D, Kuhtz-Buschbeck J, Golge M, Illert M, Deuschl G, Krack P: Subthalamic nucleus stimulation for Parkinson's disease preferentially improves akinesia of proximal arm movements compared to finger movements. Mov Disord 2003;18: 1162-1169.
Lopiano L, Torre E, Benedetti F, Bergamasco B, Perozzo P, Pollo A, Rizzone M, Tavella A, Lanotte M: Temporal changes in movement time during the switch of the stimulators in Parkinson's disease patients treated by subthalamic nucleus stimulation. Eur Neurol 2003;50:94-99.

14 Nakamura K, Christine CW, Starr PA, Marks WJ Jr: Effects of unilateral subthalamic and pallidal deep brain stimulation on fine motor functions in Parkinson's disease. Mov Disord 2007;22:619-626.

15 Kumru H, Summerfield C, Valldeoriola F, Valls-Sole J: Effects of subthalamic nucleus stimulation on characteristics of EMG activity underlying reaction time in Parkinson's disease. Mov Disord 2004;19:94-100.

16 van den Wildenberg WP, van Boxtel GJ, van der Molen MW, Bosch DA, Speelman JD, Brunia CH: Stimulation of the subthalamic region facilitates the selection and inhibition of motor responses in Parkinson's disease. J Cogn Neurosci 2006;18:626-636.
17 Rizzone M, Ferrarin M, Pedotti A, Bergamasco B, Bosticco E, Lanotte M, Perozzo P, Tavella A, Torre E, Recalcati M, Melcarne A, Lopiano L: High-frequency electrical stimulation of the subthalamic nucleus in Parkinson's disease: kinetic and kinematic gait analysis. Neurol Sci 2002;23(suppl 2): S103-S104.

18 Vaillancourt DE, Prodoehl J, Sturman MM, Bakay RA, Metman LV, Corcos DM: Effects of deep brain stimulation and medication on strength, bradykinesia, and electromyographic patterns of the ankle joint in Parkinson's disease. Mov Disord 2006;21:50-58. 